\title{
The Wanless report: engaging the NHS with patient needs?
}

Compiled by Tom Smith

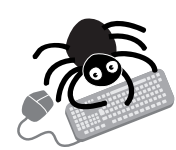

Please visit the QSHC website

(http://www.qualityhealthcare.com) for links to these articles - many to full text.

The UK Chancellor of the Exchequer Gordon Brown commissioned Derek Wanless - former CEO of Natwest Bank-to undertake a long term review of health up to the year 2022. Derek Wanless' final report relating to the future financial health of the NHS was published in March, timed deliberately to coincide with Gordon Brown's budget. The report draws on extensive consultation with academia and the health service and makes two key points: (1) the NHS needs large and sustained funding increases and (2) this investment must be carefully targeted; the second point was less widely reported. The report places particular emphasis on the need to work in different ways and for the service to engage more effectively with users and the things the public want from the health service. In the NHS of 2020 "patients are at its heart, demanding and receiving safe, high quality treatment, fast access and comfortable accommodation services. It is therefore far ahead of the present health service and a huge challenge to deliver."

$\Delta$ Wanless D. Securing our future health: taking a long-term view. The report can be accessed from the HM Treasury Internet site (http:// www.hm-treasury.gov.uk)

\section{Workforce issues}

Much of the focus of the Wanless report is on the shortage of professionals, particularly doctors and nurses. It notes the expansion of undergraduate medical places and calls for innovative solutions to the problem.

Do walk-in centres deliver improved care? - Walk-in centres are a recent innovation for the NHS and aim to meet demand for more accessible and convenient treatment. According to a new study, they provide a quality of care equivalent to standards in general practice across a range of clinical scenarios. The study used 15 actors to visit walk-in centres, general practice, and the NHS website NHS Direct, presenting different clinical scenarios in order to compare the quality of care given for the scenarios selected by the research team. Five scenarios were studied: (1) a request for postcoital contraception to assess how providers dealt with a straightforward problem; (2) a complaint of chest pain to assess the ability to exclude a potentially serious diagnosis; (3) a complaint relating to sinusitis to assess issues around antibiotic prescribing; (4) a headache complaint to assess ability to explore psychosocial issues; and (5) a scenario on asthma to assess history taking and awareness of side effects of drugs. Each role player visited a particular walk-in centre or general practice once and, because of the smaller number of sites in the research, contacted the NHS Direct sites up to three times. 91\% of consultations in walk-in centres were solely with a nurse, $96 \%$ in general practice were with a doctor, and a minority of consultations $(3 \%)$ in NHS Direct were with a call handler rather than a nurse. Compared with general practice, the walk-in centres achieved a significantly higher mean score, although there were differences between scenarios. Quality of care was better for postcoital contraception and asthma in walk-in centres, but there was little difference for sinusitis and for headache. Chest pain was treated better in general practice, though not significantly so. For each scenario, walk-in centres scored better on history taking while general practices scored marginally better on examination for chest pain and asthma. The researchers found no significant difference between quality of diagnosis, advice, and treatment between walk-in centres and general practice. When all the scenarios were combined, walk-in centres "achieved a significantly greater mean score for all items than did NHS Direct". About a quarter of consultations in walk-in centres resulted in referral compared with four-fifths in NHS Direct. Contacting NHS Direct was "sometimes time consuming and unsatisfactory. About one quarter (25 of 99) involved 'call back' (mean wait 33 minutes)." Three consultations were not completed owing to length of wait. The authors say that walk-in centres provided equivalent if not better care than both general practice and NHS Direct, performing particularly well in the asthma and contraception scenarios, though less well in examination of chest pain and diagnosis, advice and treatment of sinusitis.

- Grant C, Nicholas R, Moore L, et al. An observational study comparing quality of care in walk-in centres with general practice and NHS Direct using standardised patients. BM 2002;324:1556 (free full text article at bmj.com)

Do nurses provide equivalent care to doctors? - As nurses provide the majority of care in walk-in centres $196 \%$ in the above study), the report supports suggestions that nurses provide care of an equivalent standard to doctors. A BM paper takes a systematic review of studies (randomised controlled trials and prospective observational studies) comparing nurse practitioners and doctors working in a similar way as concurrent controls. The paper notes that several factors have led to an expansion in the role of nurses "including issues of cost, the need to increase provision of care to improve access, the availability of doctors, and the skills and expertise of nurses". This kind of change is supported strongly in the Wanless report, although an editorial in the March issue of Health Service Journal suggested that Wanless makes a number of assumptions about the future workforce. The authors say "it is important to consider whether the evidence supports the notion that nurse practitioners can substitute for doctors". The overall finding from studies incorporating patient satisfaction measures is that "patients were more satisfied with consultations with nurse practitioners than those with doctors". The paper suggests that "if patients are more satisfied with care provided by nurse practitioners, then the factors that lead to this effect should be elucidated". They suggest the reasons may be to do with "differences in training and consultation skills of nurses, patients' expectations or the extra length of time that nurses spend in consultations". These thoughts chime with evidence presented to the Wanless review: that patients judge quality of care according to the quality of softer issues around consultation. The paper concludes that further work is needed to explore a wider range of patient groups "including those with complex psychosocial problems or chronic diseases". But further research into the roles of nurse practitioners is difficult in the absence of explicit skills and experience needed at this grade, which is not clearly defined.

$\Delta$ Horrocks S, Anderson E. Salisbury C. Systematic review of whether nurse practitioners working in primary care can provide equivalent care to doctors. BM 2002;324:819-23

Are doctors produced by medical schools fit for practice? - Medical studies frequently make national headlines. A paper from the Postgraduate Medical Journal did so in May when it found "trainee doctors, many of them having just completed medical school training, have significant gaps in their knowledge and understanding of acute illness and the basic, but potentially life saving, care required to support life". The number of doctors is a key issue in the Wanless report and, in addition to questions already asked about plans to increase quantity, the paper adds others about quality and the extent to which medical schools produce doctors that are fit for practice. The results are particularly worrying, say the authors, as "most of the initial assessment and 
treatment of acutely ill ward patients is provided by trainee doctors". They say that the cause of substandard care "is inevitably multifaceted" but "basic education in acute care and the preparation of staff for clinical practice may be important determinants". The researchers received a 100\% response from 108 PRHOs from six hospitals and $77 \mathrm{SHO}$ from three to a survey assessing knowledge of airway obstruction; use of nonrebreathing oxygen masks; pulse oximetry; capillary refill time; normal minimum hourly urine output; management of the unconscious adult; survival to discharge of patients after an in-hospital cardiac arrest; and legal aspects of consent in adults.

"Many of the areas of clinical practice chosen for study by us relate specifically to abnormalities that herald clinical deterioration. Published evidence already suggests that such abnormalities that often go unnoticed are misinterpreted or ignored and that, eventually, these result in a catastrophic patient outcome. The results of our study suggest that knowledge gaps may have an important part to play in these errors and that medical school training may not prepare medical students for their forthcoming responsibilities and clinical experiences."

"Surprisingly, the performance of PRHOs and SHOs was similar, suggesting that experience gained during the pre-registration year does not necessarily correct such deficiencies."

The paper calls for "undergraduate basic skills training" and recommends that "all medical schools incorporate such training in their curriculum urgently".

A Smith GB, Poplett N. Knowledge of aspects of acute care in trainee doctors. Postgrad Med J 2002;78:335-8

\section{Engaging patients in their health care}

Another key element of the Wanless report is the need for the NHS to be more responsive to what patients want. A number of papers provide a fascinating insight into the experience of patient centredness in the NHS in terms of patient decision making, planning care with patients, and the involvement of patients and users in the development of National Service Frameworks (NSFs).

What goes through your mind when having a heart attack? - A paper from the BM helps provide "an understanding of the factors influencing the decision making process in people with symptoms of a heart attack". The study was conducted in two district general hospitals in Yorkshire serving both rural and urban populations. The participants (20 men and two women) were asked to "recount their experience of the heart attack". Participants experienced problems "identifying and labelling symptoms ... and many thought their symptoms were severe enough to be a heart attack. Instead of crushing chest pain, many attacks were reported to have had a low onset with only mild pain and breathlessness". Perception of risk is one of the key factors influencing the "time to action". Others include: how symptoms are appraised, previous experience of symptoms, beliefs about appropriate use of the NHS, and the context in which the symptoms were experienced. "Many people did not want to believe they were having a heart attack, tending to play down or ignore symptoms and wait until they became worse before seeking help". For example, patients who had changed lifestyle since a previous attack believed "this would protect them from future cardiac problems". Over $90 \%$ reported symptoms that were not similar to those experienced previously. Some who did not want to feel embarrassed by feeling "a fraud" had reported a previous false alarm and delayed a decision to seek help. Many people simply "do not want to bother the doctor". The context of the event matters: if others noticed problems, participants were more likely to seek help more quickly. The decision to seek help "is a complex interaction of knowledge and experience, beliefs, emotions, and the context of the event". The authors conclude that "no single determinant has overall primacy" and that "interventions to reduce delay should be tailored to cover the themes identified".

$\Delta$ Pattenden J, Watt I, Lewin JP, et al. Decision making processes in people with symptoms of acute myocardial infarction: qualitative study. BM 2002;324:1006-9

To what extent does co-management of care exist between doctors and patients? - Two papers take quite different approaches to the exploration of whether the extent of co-planning of care between clinician and patient is effective. Both studies relate to care plans - the first for patients with asthma and the second for expectant mothers.

Asthma care plans A group of researchers from Australia noted that meta-analysis of action plans has been associated with improved asthma outcomes. They also noted the declining use of plans "despite strong advocacy for their use and evidence of clinical benefit". They set out to understand why and to explore patients' perceptions of plans. Their conclusions suggest that plans are used in different ways: some follow the plan precisely, others modify it to their own experience, use it as a guide, or reject it in favour of their own informal plan based on personal experience rather than being regulated by the readings of a peak flow meter. A large number (33 of 64) had no action plan, in most cases because one had not been offered. Doctors perceive problems in their implementation. The study suggests that successful plans depend on genuine negotiation between doctor and patient. "We show that an iterative process encompassing experience of disease would enhance use of an action plan".

$\Delta$ Douglass J, Aroni R, Goemann D, et al. A qualitative study of action plans for asthma. BM 2002;324:1003-5

Obstetric care plans Competing demands in the clinical environment undermine the effective use of information to help patients make informed choices about their obstetric care. "Time pressures limit discussion, and choice was not available in practice". This paper examines the impact of a leaflet called Informed Choice which aims to help women influence the management of their obstetric care. Although health professionals were initially positive about the principles underpinning the leaflets, in some cases the leaflets were withheld from women because staff disagreed with the content or because they promoted choices that were not available locally. Sometimes leatlets were not given explicitly, but wrapped up in other material. One midwife told the team: "There are some ladies you don't want to be giving the leaflets to because you don't want them thinking they can have choices that aren't available. There are some women who can't read, for example ... and the young girls don't tend to be that interested." Staff sometimes expressed a strong dislike of an option covered by the leaflet to the extent that distribution was terminated. The results do not bode well for the aim of creating more openness and joint planning in care. The research team observed "a strong hierarchy within the maternity services". "The organizational and hierarchical structure of the maternity services worked against maximizing the potential of the leaflets, the relative lack of continuity of care observed . . . made it difficult for women to follow up on issues raised in a previous consultation or to initiate discussion on leaflets and other topics related to pregnancy." Choice and decision making were heavily influenced by "the pressures and norms of obstetric culture" and these "encourage informed compliance even though staff adopted the rhetoric of informed choice"

A Stapleton H, Kirkham M, Thomas G. Qualitative study of evidence based leaflets in maternity care. BM 2002;324:639 (free full text article at bmj.com)

How patient focused has the experience of developing NSFs been? - A paper from Consumer Policy Review provides a fascinating insight into "how the patients and the public, in particular hard to reach groups, have been involved in the NSFs (National Service Frameworks) to date".

The first task is to define the scope of an NSF. "There is a debate about whether NSFs are more effective when they cover a broad service area or have a narrower focus". A broad focus may affect more people but lead to vague standards. The paper suggests it depends on the area, but notes more generally that "users often complain that they are invited to contribute where the agenda has already been decided and the limits for discussion set"; they did not feel they could influence the important scoping stage. Users also felt that the NSFs were dominated by the medical and public safety agenda. Users looking at mental health and older people would rather incorporate holistic issues such as emotional, psychological and spiritual needs. All NSFs were produced with the help of External Reference Groups (ERGs), with appointments made by Ministers. Once NSFs were announced, "the various stakeholder groups start to jostle for position and some actively lobbied officials and politicians in order to be included". As opportunities to present evidence became available "membership 
of the ERG became less of an issue". Some voluntary organisations commented that some professional representatives "were seen to represent the establishment and traditional ways of working, while professionals seen as more innovative were not included. It was felt too that not all issues were represented-for example, "the ERG for older people did not include anyone from housing, public health or health promotion". Groups were not thought to use people as well as they could. "Someone from a user-led organization was, for example, out on a the working group dealing with employment issues. On another ERG a patient representative was asked to co-chair a group on technical clinical issues about which she had no knowledge or understanding."

Some of the recommendations were lost in the final report. It is suggested that the mental health NSF ERG "produced over 30 standards that were reduced to seven in the final report". Users felt the working groups were not open enough-for example, a number of what were though excellent papers were not made available. "There is sometimes a suspicion that confidentiality was used as a method of controlling user members".

"Some user-led organisations and some Department off Health officials saw voluntary organizations run by professions as an unsatisfactory proxy for real patients' and carers' voices."

"Activist members of voluntary groups are liable to be regarded as unrepresentative and therefore unable to represent the views of their peers. Similar assumptions are not made about professional members appointed to advisory committees, though the same problem might be identified, for example, where a non-practising hospital doctor attempts to represent, for example, the views of a single-handed inner city GP."

The paper makes a number of recommendations; users do not always understand their role or its limits and there should be better briefing and debriefing. "Anyone not used to operating at national level will need training, preparation and support in order to feel an equal in a group of professionals". "Finance is a barrier to participation for some people, in particular carers and people with low income". There was some inconsistency in remuneration for different groups as well as for those in the same group. Despite a fairly critical analysis "most people felt that the effort had been worthwhile and suggested three main benefits: opportunities to influence the standards; greater knowledge and understanding of the ways of the NHS; better placed to become involved in implementation". The paper suggests that effort should be put into capacity building working with particular groups or communities "to enable them to share experiences and participate in wider debates".

$\Delta$ Hogg C. National Service Frameworks: patient and public involvement. Consumer Policy Review 2002;12:49-61

T Smith

Judge Institute of Management Studies, Cambridge University, Cambridge CB2 1 1AG, UK; tabletom@btinternet.com 